\title{
Concerning de Oliveira-Junior et al. "Oncoplastic Surgery in Breast-Conserving Treatment: Patient Profile and Impact on Quality of Life." Breast Care. 2021;16(3):243-253
}

\author{
Fernando Hernanz \\ Breast Unit, Hospital Valdecilla, University of Cantabria, Santander, Spain
}

Firstly, I would like to congratulate de Oliveira-Junior et al. [1] for their article; I am fully aware of the effort they have made to conduct this study. The comparison between conventional and oncoplastic approaches in breastconserving treatment (BCT) based on patient-reported outcomes is a current issue that deserves to be discussed and addressed. After reading the work, I would like to comment on some statements and emphasize some results.

The authors wrote: "Our study has limitations primarily because it was a non-randomized study that compared clearly heterogeneous groups" [1]. That is true, but could they have carried out a randomized study? That is, could they offer only a standard lumpectomy (SL) without oncoplastic technique (oncoplastic surgery; OS) to a patient at risk of serious cosmetic sequelae? Possibly not, and the answer could be found in the following sentence: "Due to ethical issues, it is also extremely difficult to perform a randomized clinical trial that compares OS and BCT" [1].

Although it is generally accepted, and frequently written, that well-designed prospective multicenter cohort studies with propensity-matched outcomes evaluated with standard tools are needed to add more and better evidence in this field [2], one thing is clear: this scenario is highly unlikely because there are significant obstacles to overcome. For example, if the OS approach is available in the breast unit, the random selection of patients has ethical limitations: to avoid them and match the patients treated with the two approaches, they must come from different centers - some of them without oncoplastic training where only SL can be offered. In that case, at present, the problem is to find these centers, because this situation is increasingly strange.

The main conclusion of the article, "OS allowed similar aesthetic results to the classical conservative breast surgery" [1], reinforces my own experience. In my breast unit, where both approaches are available, the quality of life and satisfaction with the breasts after OS or SL evaluated by the BREAST-Q (BCT module, Spanish version) were similar [3]. A plausible explanation for these results is that the OS approach improves the outcome of the SL cohort by avoiding treating patients at risk of cosmetic sequelae if they are treated with a simple lumpectomy. In my opinion, the authors made the same bias in the selection of patients, since those who underwent OS were younger and had large tumors; were these patients at risk of having poor cosmetic results with SL?

Another important point mentioned concerns the indication for OS. In this study, the main factors related to the indication for OS are described and the authors pointed out that there are no clear criteria that define which patients should undergo OS. Likewise, they commented that as surgeons gain experience with different OS techniques, the indication for BCT-OS increases and is associated not only with tumor aspects but also with aesthetic results, thus increasing the rate of bilateral surgery.

Bearing these considerations in mind, I propose that an OS approach should be appreciated for its side effects (externalities), such as a reduction in the percentage of reoperation for affected surgical margins, the increment of the percentage of BCT avoiding mastectomies with difficult breast reconstruction (extreme oncoplastic ap- 
proach), and the improvement of cosmetic results of a conventional lumpectomy, minimizing the risk of severe aesthetic sequelae. Therefore, instead of comparing SL with OS or evaluating OS separately, it would be more purposive to determine a "standard range of percentage of oncoplastic conserving approach," in which patients are treated correctly with simple lumpectomy, reserving more complex and extensive surgery for patients who really need it and avoiding overtreatment on the condition that this mix of BCT obtains good outcomes.

I would like to highlight a very important result that may go unnoticed in this series of 297 patients with $24 \%$ OS, namely the cosmetic outcomes, which are closely re- lated to surgical technique; they were evaluated by the BCCT core software and were good or excellent in 30.0\% of the patients and reasonable in $45.5 \%$. These data are a first step in achieving the aforementioned objective.

\section{Conflict of Interest Statement}

Fernando Hernanz has no conflicts of interest to declare.

\section{Funding Sources}

This letter received no funding.

\section{References}

1 de Oliveira-Junior I, de Araujo da Silva I, Brandini da Silva FC, da Silva JJ, Sarri AJ, Paiva $\mathrm{CE}$, et al. Oncoplastic surgery in breastconserving treatment: patient profile and impact on quality of life. Breast Care. 2021;16(3): 243-53.

2 Rocco N, Catanuto G, Cinquini M, Audretsch W, Benson J, Criscitiello C, et al. Should oncoplastic breast conserving surgery be used for the treatment of early stage breast cancer? Using the GRADE approach for development of clinical recommendations. Breast. 2021 Jun;57:25-35.

3 Hernanz F. Comments on patient-reported outcome after oncoplastic breast surgery compared with conventional breast-conserving surgery in breast cancer. Breast Cancer Res Treat. 2021 Jan;185(2):529-30. 